\title{
Registros de Notificação Compulsória de Violência Envolvendo Crianças e Adolescentes
}

\author{
Pâmela Kurtz Cezar \\ Universidade Federal de Santa Maria, RS, Brasil. $\quad$ Pontifícia Universidade Católica de São Paulo, SP, Brasil. \\ Everley Rosane Goetz \\ Universidade Federal de Santa Catarina, SC, Brasil.
}

\begin{abstract}
Resumo: Este estudo objetivou apresentar dados epidemiológicos de notificações compulsórias da violência na faixa etária da infância e da adolescência, no período de 2009 a 2013, ocorridos e notificados em uma cidade do interior do estado do RS, identificando o sexo das vítimas, o tipo de violência sofrida, o local onde ocorreu o fato e o tipo de vínculo com o agressor. Para isso, realizou-se uma pesquisa epidemiológica descritiva e documental com dados secundários do Sistema de Informação de Agravos de Notificação, analisados conforme estatística descritiva. Os resultados indicaram que o sexo feminino predominou no número de vítimas, a violência física foi a mais notificada, a residência foi o local onde mais ocorreram as violências, tendo a mãe e o pai como os principais agressores. Conclui-se, a partir desses resultados, que a notificação compulsória da violência é um desafio ao campo da saúde e que esse procedimento pode ser um instrumento de proteção à infância e a adolescência, quando integrado a outras práticas de cuidado.
\end{abstract}

Palavras-chave: Violência, Criança, Adolescente, Notificação de Abuso.

\section{Records of Compulsory Notification of Violence Against Children and Adolescents}

\begin{abstract}
This study aims to present epidemiological data of compulsory notification of violence against children and adolescents from 2009 to 2013 occurred and reported in a countryside city in the state of Rio Grande do Sul, identifying the gender of the victims, the type of violence suffered, the place where it happened and the type of relationship with the offender. A descriptive and documentary epidemiological research was performed, using secondary data from the Notifiable Diseases Information System that were then analyzed according to descriptive statistics. The results indicated that the victims were predominantly female, physical violence was the most commonly reported, the residence was the place where most of the violence occurred and the mother and the father were the biggest offenders. In conclusion, the compulsory notification of violence is a challenge to the public health field and such procedure could be an instrument for protecting children and adolescents when integrated to other healthcare practices.
\end{abstract}

Keywords: Violence, Child, Adolescent, Abuse Notification. 


\section{Registros de Notificación Obligatoria de Violencia contra Niños y Adolescentes}

Resumen: El estudio tiene como objetivo presentar datos epidemiológicos de notificaciones obligatorias de violencia en el grupo etario de la infancia y adolescencia, en el período de 2009 a 2013, ocurridos y notificados en una ciudad del interior del estado de RS, identificando el sexo de las víctimas, el tipo de violencia sufrida, la ubicación donde ocurrió el episodio, el hecho y el tipo de vínculo con el agresor. Fue realizada una investigación epidemiológica descriptiva y documental, utilizando datos secundarios del Sistema de Información de Agravios de Notificación, que fueron luego analizados conforme la estadística descriptiva. Los resultados indicaron que el sexo femenino predominó en el número de víctimas, la violencia física fue la más notificada, la residencia fue el lugar donde más ocurrieron las violencias, siendo la madre y el padre los mayores agresores. Se concluyó que la notificación compulsoria de la violencia es un desafío para el campo de la salud y que ese procedimiento puede ser un instrumento de protección para la infancia y la adolescencia, al estar integrado a otras prácticas de cuidado.

Palabras clave: Violencia, Niños, Adolescente, Notificación de Abuso.

\section{Introdução}

A violência é um fenômeno que acompanha a trajetória humana desde os mais antigos registros. São incontáveis as maneiras pelas quais a violência se expressa, podendo ainda se adaptar às peculiaridades culturais e às características de cada momento histórico (Ariès, 1981; Assis, 1994; Minayo, \& Souza, 1999). Embora o entendimento do que é violência tenha se transformado nas últimas décadas, o que temos na atualidade não é necessariamente uma redução ou um aumento da violência envolvendo a infância e a adolescência, mas uma mudança de discurso, uma tomada de consciência acerca da importância de proteger essa parcela da população (Eliachef, 2007; Santos 2014).

A partir de 1980, a luta pelos direitos humanos e pelos diretos das crianças e dos adolescentes ganhou força expressiva. Nesse período, houve inúmeros movimentos da sociedade civil e de setores do Estado, somados a acontecimentos internacionais como a Convenção das Nações Unidas pelos Direitos da Criança, em 1989 (Faleiros, \& Faleiros, 2008; Santos, 2014).

No Brasil, as medidas mais significativas foram a inclusão do artigo 227 na Constituição Federal de 1988 e a criação do Estatuto da Criança e do Adolescente (ECA) em 1990. Nesse sentido, o artigo 227 da Constituição Federal expõe que é dever da família, da sociedade e do Estado assegurar com prioridade à criança e ao adolescente o direito a uma vida digna, colocando-os a salvo de toda forma de negligência, discriminação, exploração, violência, crueldade e opressão (Senado Federal, 1988).

Destaca-se que as décadas de 1980 e 1990 também foram importantes para a constituição do Sistema Único de Saúde (SUS), bem como para a inserção da Psicologia no campo da saúde pública (Cezar, Rodrigues, \& Arpini, 2015). Por sua vez, a inclusão da violência na pauta da saúde pública brasileira ocorreu de maneira lenta e gradual, visto que historicamente o fenômeno foi abordado, sobretudo, no âmbito do direito criminal e da segurança pública. As ações no campo da saúde restringiram-se, num primeiro momento, a intervenções curativas, na qual os profissionais de saúde atuavam essencialmente como reparadores das lesões causadas pela violência (Minayo, 1994; 2006; Minayo, \& Souza, 1999).

A partir da década de 1990, a temática da violência ganhou mais espaço no campo da saúde. No final da década, uma política pública nacional começou a se delinear com a intenção de incluir no âmbito do SUS ações direcionadas para seu enfrentamento. Pois, mesmo que a violência não seja questão específica da saúde, transformou-se em assunto para esse setor devido aos impactos que ocasiona na saúde individual e coletiva da população (Minayo, 1994; 2006). 
Com o advento do ECA em 1990, os profissionais de saúde foram convocados a intervir de maneira diferenciada nas ocorrências de violência envolvendo crianças e adolescentes. Somente cuidar das lesões causadas por esse fenômeno não era suficiente, os profissionais de saúde tornaram-se também solicitados a notificar as situações de violência. $\mathrm{O}$ artigo 13 do ECA define que os casos de suspeita ou confirmação de maus-tratos contra crianças ou adolescentes devem ser obrigatoriamente comunicados ao Conselho Tutelar da respectiva localidade, sem prejuízo de outras providências legais (Brasil, 1990).

Diante disso, o notificante é considerado um ator fundamental na rede de proteção à infância e a adolescência, pois é a pessoa que pode tornar pública a violência (Garbin, Dias, Rovida \& Garbin, 2015; Gonçalves, Ferreira, \& Marques, 1999). Portanto, a notificação torna-se um valioso instrumento promotor de política pública, visto que ajuda a dimensionar a magnitude da violência e a definir, mais adequadamente, a necessidade de investimentos dos órgãos públicos em serviços e intervenções direcionadas ao enfrentamento do fenômeno (Assis, Avanci, Pesce, Pires, \& Gomes, 2012; Brasil, 2011a; Gonçalves, \& Ferreira, 2002; Santos, Ippolito, \& Magalhães, 2014).

Em 2001, o Ministério da Saúde, reconhecendo o impacto social e econômico das violências, implantou a Política Nacional de Redução da Morbimortalidade por acidentes e violências, por meio da Portaria $n^{\circ}$ 737. Em 2006, foi publicada a Portaria no 1.356, implantando-se o Sistema de Vigilância de Violências e Acidentes (VIVA). Esse sistema tinha a finalidade de obter e divulgar dados epidemiológicos sobre violências e acidentes no Brasil, sendo estruturado em dois componentes: Vigilância Contínua e Vigilância Sentinela. O primeiro captava dados de violência doméstica, sexual e/ou outras em serviços de saúde por meio do preenchimento de uma ficha denominada "Ficha de notificação/investigação individual de violência doméstica, sexual e/ou outras violências". O segundo componente, VIVA Sentinela ou Vigilância por Inquérito, constituiu-se em uma modalidade de vigilância pontual realizada com base nas notificações de violência em serviços sentinelas de urgência e emergência (Assis et al., 2012; Brasil, 2008; 2011a).

Em 2008, uma parceria entre o Departamento de Informática do SUS, Gerência Técnica do Sistema de Informação de Agravos de Notificação (Sinan) e Área Técnica de Vigilância e Prevenção de Vio- lências e Acidentes, desenvolveu um aplicativo de entrada de dados no Sinan referente às violências (Brasil, 2011a). Ademais, a partir de 2009, o "Módulo Violência” foi integrado ao Sinan-Net, para que fosse possível uniformizar e dimensionar a magnitude das violências em todo o país, tendo em vista que, anteriormente ao Sinan, os municípios adotavam fichas próprias para as notificações das violências, gerando informações heterogêneas e desarticuladas (Assis et al., 2012).

No entanto, com a necessidade de padronizar os métodos referentes à notificação compulsória e à vigilância em saúde, o Ministério da Saúde emitiu, em 2011, a Portaria no 104, reforçando a notificação da violência como obrigatória a todos os profissionais de saúde, responsabilizando-os quanto ao preenchimento da ficha do Sinan. Essa Portaria incluiu a violência na lista das doenças, agravos e eventos em saúde pública de notificação compulsória em todo o território nacional (Brasil, 2011b).

A Ficha de Notificação Compulsória da Violência é composta por variáveis e categorias que englobam as violências autoprovocadas e as violências interpessoais. Por meio de seu preenchimento, torna-se possível revelar a magnitude da violência, sua tipologia, gravidade, perfil das pessoas envolvidas, sejam elas vítimas e/ou autores da agressão, localização de ocorrência, entre outras características dos eventos violentos. Assim, a notificação nos serviços de saúde deve ser realizada sempre que houver atendimento de uma situação suspeita ou confirmada de violência. Excepcionalmente nos casos de violência contra crianças e adolescentes, uma comunicação, relatório impresso e/ou uma cópia da ficha de notificação deve ser encaminhada ao Conselho Tutelar ou a autoridades competentes, conforme estabelecido no ECA (Brasil, 1990; 2011a).

No ano de 2014, a publicação da Portaria no 1.271 estabeleceu que a notificação compulsória deveria ser realizada de maneira imediata ou semanal. A violência doméstica e outras violências tornaram-se eventos de notificação semanal e a violência sexual e a tentativa de suicídio, eventos de notificação imediata (Brasil, 2014). Neste mesmo ano, o Ministério da Saúde publicou outra versão da ficha de notificação, denominada Ficha de Notificação/Investigação individual - Violência interpessoal/autoprovocada.

Considerando o exposto, este estudo tem como objetivo apresentar os dados epidemiológicos das noti- 
ficações compulsórias da violência na faixa etária da infância e da adolescência no período de 2009 a 2013 ocorridos e notificados em uma cidade do interior do estado do Rio Grande do Sul, identificando o sexo das vítimas, o tipo de violência sofrida, o local onde ocorreu o fato e o tipo de vínculo com o agressor. Com o estudo, pretende-se também subsidiar o trabalho dos profissionais envolvidos com a temática da violência na infância e na adolescência por meio do conhecimento dos dados epidemiológicos, para que seja possível repensar e ampliar as práticas de cuidado.

\section{Metodologia}

Este estudo refere-se a uma pesquisa quantitativa, epidemiológica descritiva e documental. Por documento, entende-se toda fonte de dados já existente, mas que não estão ultrapassados e, portanto, representam recursos vivos de informação (Laville, \& Dionne, 1999). Os documentos podem estar na forma de texto ou de arquivo eletrônico, como num banco de dados, por exemplo, sendo capazes de proporcionar informações em nível pessoal ou institucional (Flick, 2009).

Desse modo, a pesquisa documental compreendeu a utilização de dados secundários, disponíveis publicamente, no Banco de dados do Sinan-Net: violência doméstica, sexual e/ou outras, por meio do acesso aos dados da Vigilância Epidemiológica TabNet/RS (Rio Grande do Sul, 2014). Durante o mês de janeiro de 2014, foi realizada uma busca e seleção de dados referentes à notificação compulsória da violência em uma cidade de médio porte do interior do estado do Rio Grande do Sul, especificando que este foi o município de notificação, de residência e de ocorrência dos fatos.

As variáveis utilizadas na busca dos documentos foram: (a) o total de registros de notificação compulsória; (b) o sexo das vítimas; (c) o tipo de violência sofrida; (d) o local onde ocorreu o fato; e (e) o tipo de vínculo com o agressor. Os dados epidemiológicos obtidos junto ao TabNet/RS corresponderam ao período de 2009 a 2013. Dessa forma, os dados apresentados referem-se ao preenchimento da Ficha de notificação/investigação individual de violência doméstica, sexual e/ou outras violências do Sinan - versão 2008. Como o estudo teve por foco a infância e a adolescência, foram incluídas na busca vítimas de violência na faixa etária compreendida entre 0 e 19 anos, mas também apresentados resultados separadamente por faixa etária de 0 a 9 anos e de 10 a 19 anos, para verificação de possíveis diferenciações e comparações.

$\mathrm{Na}$ análise dos dados, utilizou-se da estatística descritiva com demonstração das frequências relativas e absolutas, com exposição dos resultados sintetizados por meio de figuras. Inicialmente, na seção dos resultados os dados referentes às notificações compulsórias da violência (NCV), são apresentados a partir de figuras e posteriormente na discussão esses dados são discutidos com outros achados científicos.

\section{Resultados}

Pelo levantamento dos dados epidemiológicos do Sinan referentes às NCV, na cidade investigada, foram encontrados 316 documentos, indicativos de que, no período compreendido entre 2009 a 2013, houve 316 registros de notificação envolvendo a faixa etária entre 0 e 19 anos (dados por residência, ocorrência e notificação). No ano de 2009, registrou-se apenas uma notificação; em 2010, foram 10; em 2011, foram 51; em 2012, foram 140; e, em 2013, notificaram-se 114 casos, conforme apresentado na Figura 1.

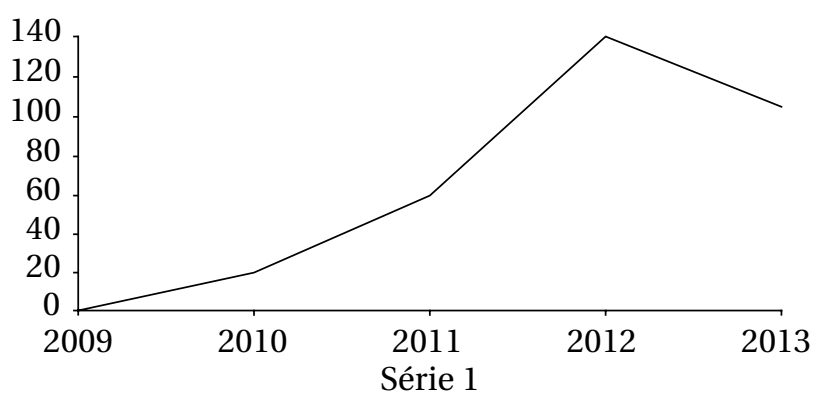

Fonte: Sinan/TabNet-RS (2009-2013)

\section{Figura 1}

Distribuição do número de NCV na faixa etária entre 0 e 19 anos, no período entre 2009 a 2013.

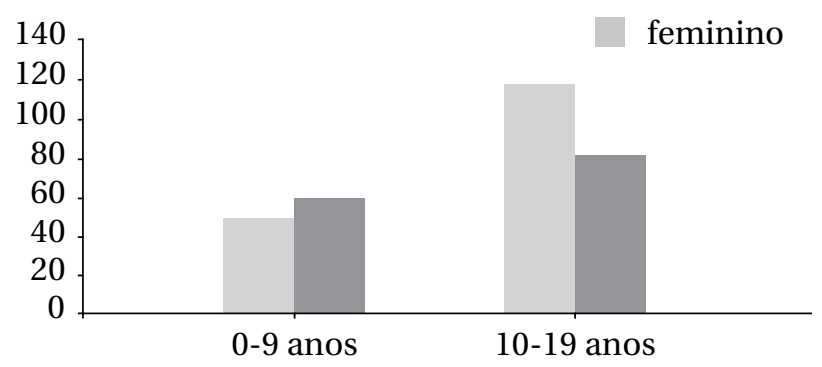

Fonte: Sinan/TabNet-RS (2009-2013)

Figura 2

Distribuição dos registros de NCV no período entre 2009 a 2013, de acordo com o sexo e a faixa etária. 
Esses resultados parecem indicar que, na medida em que foi se desenvolvendo um processo que torna a notificação uma ação relevante nos serviços de saúde, o número de registros foi sensivelmente sendo ampliado ano após ano, dando mais visibilidade a um fenômeno que historicamente ficou silenciado. Ao notificar, é possível conhecer os principais aspectos que se encontram envolvidos, tendo elementos para trabalhar a promoção, prevenção e tratamento dos eventos violentos, envolvendo os profissionais de saúde dos diferentes níveis de atenção. Os psicólogos têm, sem dúvida, um grande desafio a enfrentar a partir desses resultados. Aspectos como cuidados éticos, sigilo e registro de informação são elementos que se colocam com grandes desafios a partir da notificação.

Do total das 316 notificações entre 2009 a 2013, aproximadamente $57 \%$ foram contra o sexo feminino e o restante (43\%) contra o masculino. Houve 106 registros de NCV na faixa etária entre 0 e 9 anos, entre os quais 56 tiveram como vítimas as meninas e 50, os meninos. Em relação à faixa etária entre 10 e 19 anos, houve 210 registros, sendo 123 de violência contra meninas e 87 contra meninos, conforme observado na Figura 2.

Observa-se que o sexo feminino predominou no número de vítimas de violência, em especial na adolescência. No que se refere aos tipos de violência, as notificações revelaram que, na faixa etária entre 0 e 9 anos, predominou a negligência/abandono com 41 registros, acompanhada da violência psicológica/moral com 34, da violência sexual com 31 e da violência física com 29 registros. Na faixa etária entre 10 e 19 anos, prevaleceu a violência física com 98 registros, seguida da violência sexual com 60, de outros tipos de violência com 57 e da lesão autoprovocada com 56 notificações, conforme demonstrado na Figura 3.

Identifica-se, conforme exposto na Figura 3, que na infância predominaram as situações de negligência/abandono e na adolescência prevaleceu à violência física. Contudo, destaca-se, nessa fase, a violência autoprovocada. No que diz respeito ao local onde ocorreram as violências, identificou-se que mais de $68 \%$ dos casos ocorreram na própria residência das vítimas. Na faixa etária entre 0 e 9 anos, houve 106 registros de $\mathrm{NCV}$, dos quais 93 tiveram como local de ocorrência a residência. Na faixa etária entre 10 e 19 anos, das 210 NCV, 122 ocorreram na residência, seguido de 49 registros de ocorrência em via pública, conforme apresentado na Figura 4.

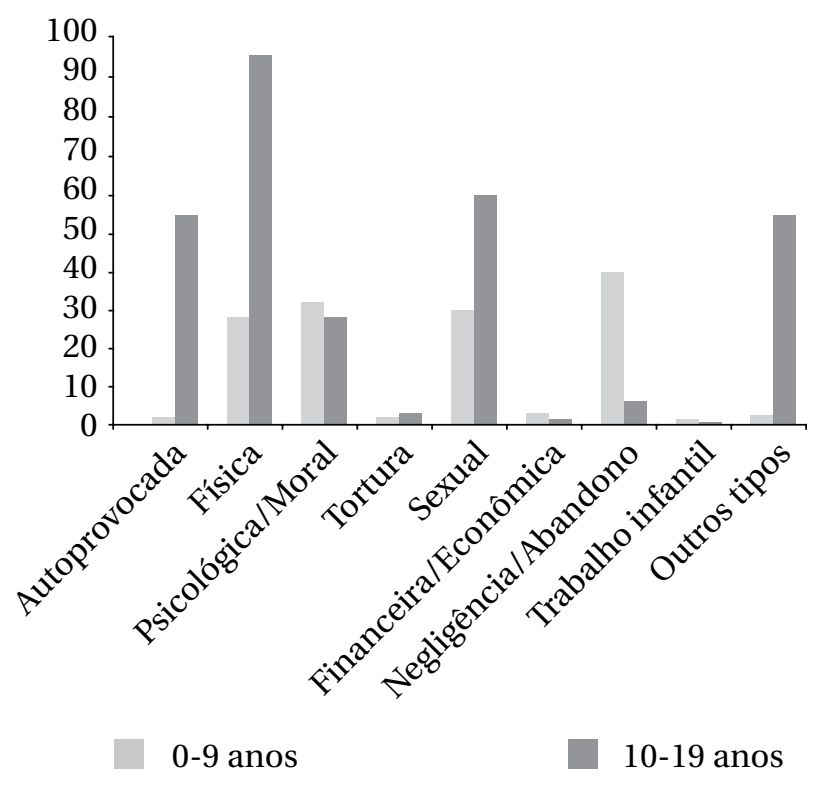

Fonte: Sinan/TabNet-RS (2009-2013)

Figura 3

Distribuição dos tipos de violências notificadas por faixa etária no período de 2009 a 2013.

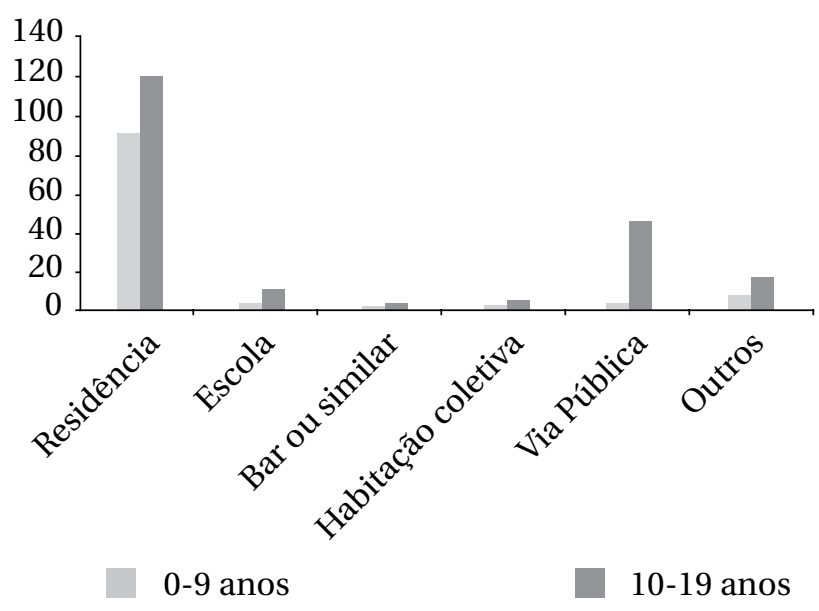

Fonte: Sinan/TabNet-RS (2009-2013)

Figura 4

Distribuição dos locais de ocorrência de violência por faixa etária no período de 2009 a 2013.

Quanto ao tipo de vínculo da vítima com o agressor, observou-se que, na faixa etária entre 0 e 9 anos, a mãe apareceu como a maior agressora com 52 registros, seguida pelo pai com 32. Na faixa etária entre $10 \mathrm{e}$ 19 anos, prevaleceu a própria pessoa com 58 registros, conforme Figura 5.

Observa-se, por meio das Figuras 4 e 5, que a residência predominou como o local de maior ocorrência 


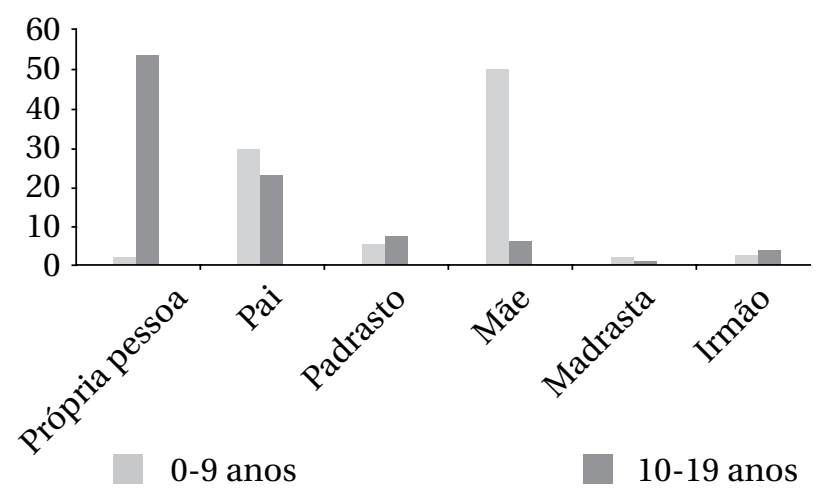

Fonte: Sinan/TabNet-RS (2009-2013)

Figura 5

Distribuição dos tipos de vínculo das vítimas com os agressores, por faixa etária no período de 2009 a 2013.

das violências que afetam crianças e adolescentes na cidade investigada. Em relação aos autores da agressão, destacam-se a mãe e o pai. Contudo, na faixa etária da adolescência, prevaleceu a própria pessoa como autora da agressão (lesão autoprovocada).

\section{Discussão}

Os dados epidemiológicos das NCV referentes à cidade investigada indicaram que, no período entre 2009 e 2013, os registros aumentaram, em especial a partir de 2011, conforme demonstrado na Figura 1.

Tal fato pode ser atribuído à emissão da Portaria $n^{\circ} 104$ em 2011, que tornou a violência um evento de notificação compulsória em todo o país, na qual os profissionais de saúde foram convocados a fazer o preenchimento da Ficha de Notificação sempre que houvesse $o$ atendimento de uma situação confirmada ou suspeita de violência (Brasil, 2011b). Outro fator que pode contribuir para elevar os índices de NCV é a Portaria no 1.271 de 2014, tendo em vista que, a partir desta publicação, as situações de violência sexual e tentativa de suicídio passam a ser de notificação obrigatória em até $24 \mathrm{~h}$ do conhecimento do fato e os demais tipos de violência devem ser notificados em até uma semana (Brasil, 2014).

Os dados apresentados na Figura 2 demonstraram o sexo feminino como maior vítima das violências na faixa etária da infância e da adolescência, com predominância na faixa etária entre 10 e 19 anos. Em relação aos tipos de violências notificadas, a violência física apareceu em primeiro lugar considerando as idades entre 0 e 19 anos. Na faixa etária entre 0 e 9 anos, predominaram as notificações de negligência/abandono. Na faixa etária entre 10 e 19 anos, prevaleceu a violência física, seguida da violência sexual, com destaque para as lesões autoprovocadas, conforme Figura 3.

A residência apareceu como local onde mais foram notificadas as violências que envolveram a infância e a adolescência, seguida da via pública, conforme observado na Figura 4. Quanto ao tipo de vínculo da vítima com o agressor, identificou-se a mãe e o pai como os maiores agressores. Na faixa etária entre 0 e 9 anos, predominou a mãe seguida pelo pai e, na faixa etária entre 10 e 19 anos, predominou a própria pessoa seguida pelo pai, segundo dados da Figura 5 .

Nessa perspectiva, um estudo realizado no TabNet-RS, considerando a faixa etária entre $10 \mathrm{e}$ 19 anos, identificou que nos anos de 2009 a 2010 as violências notificadas no estado do RS foram perpetradas sobretudo contra o sexo feminino. Em 2009, prevaleceram os registros de violência sexual contra o sexo feminino e de violência física contra o masculino. Em 2010, a violência física predominou em ambos os sexos, contudo os registros de violência sexual seguiam apontando as meninas como as maiores vítimas. Em relação ao vínculo da vítima com o agressor, o estudo mostrou que, na faixa etária de 10 a 14 anos, o pai e a mãe apareceram como os principais agressores (Zanatta, Pai, Resta, Argenta, \& Motta, 2012).

Uma pesquisa realizada na Região Metropolitana de Porto Alegre/RS investigou 1.754 registros de crianças e adolescentes, na faixa etária entre 0 e 14 anos, que foram vítimas de violência entre 1997 e 1998. Foram consultadas 75 instituições que realizavam atendimento a crianças e adolescentes, como Conselhos Tutelares, Casas de Passagem, Hospitais, Órgãos do Ministério Público, entre outros. Os resultados desta investigação apontaram que, em relação à violência sexual, $79,4 \%$ das vítimas eram meninas. O local de ocorrência dessas violências foi, em $65,7 \%$ dos casos, na residência da vítima (Kristensen, Oliveira, \& Flores, 1999).

A análise de todos os processos de violência sexual ajuizados pelas Promotorias Especializadas na Infância e na Juventude de Porto Alegre/RS e Ministério Público Estadual do Rio Grande do Sul durante 1992 e 1998, apontou que as crianças e os adolescentes vítimas de violência sexual eram do sexo feminino em $80,9 \%$ dos casos. No que diz respeito ao local de ocorrência da violência, observou-se que, em $66,7 \%$ dos casos, o abuso 
aconteceu na casa da vítima. Além disso, o agressor era, em 98,8\% dos casos, do sexo masculino e possuía vínculos afetivos e de confiança com a vítima. O pai apareceu como agressor em $57,4 \%$ das ocorrências, seguido pelo padrasto em 37,2\% (Habigzang, Koller, Azevedo, \& Machado, 2005).

Outra pesquisa, realizada por meio do acesso as fichas de atendimento no período de 2002 a 2006 no Centro de Referência no Atendimento Infanto-Juvenil em Porto Alegre, identificou que $75,0 \%$ das vítimas de violência eram do sexo feminino. Os registros de 2006 demonstraram que $82,5 \%$ dos casos notificados referiram-se à violência sexual. Desses abusos, 59,3\% foi caracterizado como intrafamiliar, nos quais os padrastos e os pais apareceram como os maiores abusadores (Pelisoli, Pires, Almeida, \& Dell'Aglio, 2010).

Estudos realizados no Brasil, em diversas cidades, setores e serviços têm apontado as violências sexual, física, psicológica e a negligência como as violências que mais afetam as crianças e os adolescentes (Apostólico, Nóbrega, Guedes, Fonseca, \& Egry, 2012; Assis et al., 2012; Brasil, 2009; Brito, Zanetta, Mendonça, Barison, \& Andrade, 2005; Costa et al., 2007; Veloso, Magalhães, Dell'Aglio, Cabral, \& Gomes, 2013). Pesquisas que utilizam o Sinan têm evidenciado que a residência foi o local onde mais aconteceram as violências na faixa etária da infância e da adolescência. Somado a isso vem o fato de que o pai, a mãe, demais familiares ou conhecidos serem os maiores agressores e o sexo feminino a maior vítima (Assis et al., 2012; Brasil, 2008; Veloso et al., 2013; Waiselfisz, 2012).

Outrossim, o que se destacou na cidade investigada foi o alto índice de notificações de violências autoprovocadas na faixa etária entre 10 e 19 anos. Essa violência é caracterizada por situações em que a pessoa atendida/vítima provocou agressão contra si mesma, englobando as tentativas de suicídio e o suicídio. A Organização Mundial da Saúde apresenta que na faixa etária entre 15 e 35 anos o suicídio está entre as três maiores causas de morte. O Brasil é considerado um país com baixas taxas de suicídio, contudo alguns estados brasileiros apresentam índices equivalentes a países com frequências consideradas de média a elevada, sendo o RS o estado com taxas mais altas de suicídio no país (Brasil, 2006).

Os dados epidemiológicos referentes às lesões autoprovocadas na cidade investigada diferem de outros estudos que utilizam o Sinan como fonte de dados. Esta questão merece atenção e medidas de intervenção por parte dos gestores, profissionais da saúde e da rede de proteção, bem como maior investigação acerca dos motivos pelos quais os adolescentes, em especial, têm cometido atos de agressão contra si mesmo. Neste aspecto, destaca-se a importância da Psicologia, tanto no atendimento das vítimas/autores das lesões autoprovocadas, como na qualificação dos demais profissionais para a possível detecção precoce dessas ocorrências.

Nesse sentido, um estudo realizado nos meses de janeiro a junho de 2004, na Delegacia de Proteção à Criança e ao Adolescente (DPCA) da mesma cidade deste estudo, demonstrou que houve um total de 1.234 registros, dos quais 455 casos envolveram 550 crianças e adolescentes como vítimas de violência. As violências mais notificadas foram lesão corporal, outras violências e ameaça. Identificou-se que o perfil predominante das vítimas era do sexo feminino, na faixa etária entre 13 e 18 anos. Quanto ao tipo de vínculo com o agressor, observou-se que a maior parte dos registros indicava que não havia relação entre vítima/agressor. Posteriormente, apareceu o pai e depois outro familiar. Quanto ao local de ocorrência das violências, a casa apareceu em primeiro lugar, seguida da rua (Arpini, \& Silva, 2006).

Percebe-se que os registros realizados num período de seis meses na DPCA superam expressivamente o número de registros do Sinan num período de cinco anos de NCV nessa cidade. A relação vítima/agressor também difere, pois na DPCA predominaram os registros em que não existia relação vítima/agressor. Nos registros do Sinan, identificou-se que prevaleceram as situações nas quais os agressores possuíam vínculo com a vítima, como mães e pais das crianças e/ou adolescentes. Por outro lado, tanto na DPCA como no Sinan, a violência física seguiu como a mais notificada, permaneceu o sexo feminino como a maior vítima e a residência como o local onde mais aconteceram as violências. Destaca-se ainda que na lista dos notificantes da DPCA os serviços de saúde sequer foram citados como autores da notificação (Arpini, \& Silva, 2006).

Por sua vez, embora os registros de notificação compulsória da violência, na faixa etária entre 0 e 19 anos, tenham aumentado a partir de 2011 na cidade investigada, os números do Sinan também indicam uma subnotificação da violência envolvendo a infância e a adolescência. Tal fato pode ser constatado, por meio da comparação com os registros na DPCA, somado ao fato que essa cidade possui popula- 
ção estimada de aproximadamente 280 mil habitantes (IBGE, 2014) e apresentou apenas 316 registros, num período de cinco anos de notificações no Sinan.

A esse respeito, um estudo com foco nas notificações de violência contra adolescentes no Rio Grande do Sul, realizado no TabNet-RS, constatou que, em 2009, houve poucos registros de NCV no estado. Do total de Coordenadorias Regionais de Saúde, 13 não apresentaram nenhuma notificação e seis tiveram números baixos de registros. Já em 2010 todas as Coordenadorias passaram a notificar a violência (Zanatta et al., 2012).

Por sua vez, uma pesquisa realizada no Sinan-Net, em 2010, acerca das notificações de violência doméstica, sexual e outras perpetradas contra crianças no Brasil, identificou que Mato Grosso do Sul, Rio Grande do Sul, Roraima e São Paulo foram os estados com maiores taxas de notificação de violência na infância. Além disso, o Rio Grande do Sul apresentou um total de 26, 4\% de municípios notificantes e apareceu como o segundo estado do Brasil com o maior número de unidades de saúde notificantes. Contudo, embora o estado do RS tenha se destacado positivamente em relação aos registros NCV, o percentual de municípios notificantes em 2010 indicava que a maioria das cidades gaúchas ainda não estava realizando tal procedimento (Assis et al., 2012).

Outra porta de entrada para a comunicação das violências é o Disque Denúncia Nacional, atualmente conhecido por Disque 100. Os dados do Disque 100, referentes ao período entre maio de 2003 e maio de 2010, indicaram que este sistema realizou mais de 2 milhões de atendimentos em todo o Brasil, das quais 125 mil denúncias foram recebidas e encaminhadas. Desse total, 16.008 foram na região Sul. Por sua vez, no ranking das unidades federativas em relação ao número de denúncias, identificou-se que o estado do RS não apareceu na lista dos dez estados com maior número de denúncias no período investigado (Moreira, Sousa, \& Silva, 2013).

Diante disso, torna-se interessante conhecer que estratégias os municípios/estados com maiores índices de NCV têm utilizado para atingir esses resultados, bem como identificar quais atitudes os municípios/estados que apresentam poucos registros de NCV têm tido em relação aos dados epidemiológicos, no sentido de problematizá-los e buscar mecanismos de enfrentamento. Tais investigações são necessárias tendo em vista que um baixo índice de notificações não significa que o município não apresenta violência contra crianças e adolescentes.
Estudos têm apresentado que as dificuldades no processo de notificação da violência podem estar relacionadas a diversos fatores, como por exemplo, pouco preparo profissional para diagnosticar e intervir nas situações de violência, fragilidades na estrutura e organização dos serviços, falta de credibilidade na rede de proteção e receios quanto aos procedimentos legais que poderiam advir da notificação (Arpini, Soares, Bertê, \& Dal Forno, 2008; Gonçalves, \& Ferreira, 2002; Lobato, Moraes, \& Nascimento, 2012).

Identificam-se inúmeros desafios ao campo da saúde que perpassam a qualificação e o suporte aos profissionais diante das situações de violência na infância e a adolescência. Os profissionais precisam sentir-se seguros e amparados para realizar a notificação e, além disso, visualizarem os resultados de sua ação. Pois, embora a notificação possa não ter um efeito transformador da realidade imediata, ela certamente é um instrumento que permite dar visibilidade às manifestações da violência, dimensionando a intensidade e as características do fenômeno.

Uma das maneiras para dar visibilidade à violência nos serviços de saúde é proporcionar qualificação aos profissionais quanto à notificação compulsória (Assis et al., 2012; Garbin et al., 2011). Assim, a violência poderá ganhar reconhecimento e o processo de notificação desempenhar efetivamente a função de descrever o perfil epidemiológico da violência, fornecendo informações para o planejamento de políticas públicas e intervenções condizentes com as realidades locais (Deslandes, Mendes, Lima, \& Campos, 2011; Garbin et al., 2011).

No entanto, é preciso estar atento para que os indicadores epidemiológicos fornecidos pelas notificações não sejam percebidos apenas como dados estatísticos. É necessário que se faça a problematização da informação por meio de discussões fundamentadas em interface com o contexto social e as demais relações que permeiam as situações de violência (Arpini, et al., 2008; Galvão, \& Dimenstein, 2009).

É preciso também questionar acerca do destino dos dados epidemiológicos. As gestões em nível municipal, estadual e/ou federal têm utilizado esses dados para planejar intervenções de enfrentamento da violência? Quem tem acesso a tais registros? Quais os motivos da subnotificação? Estas questões precisam fazer parte do cotidiano dos serviços de saúde para que a violência possa ser mais adequadamente compreendida e enfrentada. 
Mesmo que os dados epidemiológicos das NCV que envolvem as crianças e os adolescentes possam indicar uma subnotificação da violência, é necessário problematizar os dados que estão registrados no Sinan. Esses dados revelam, por exemplo, que é preciso investir no cuidado às famílias, uma vez que a residência tem sido o local de maior ocorrência das violências aliado ao fato da mãe e do pai se apresentarem como os maiores agressores.

Torna-se necessário destacar que o perfil da violência identificado neste estudo e corroborado com outras investigações tem revelado que a família pode ser, em inúmeras situações, um fator de risco ao desenvolvimento das crianças e dos adolescentes. A compreensão idealizada de família e do ambiente doméstico, na qual as crianças e os adolescentes que estão em casa com seus pais estão protegidos e resguardados da violência torna-se, em diversas situações, uma inverdade. É preciso haver o entendimento de que a violência doméstica/intrafamiliar contra crianças e adolescentes, em suas variadas manifestações - negligência, violência física, psicológica, sexual - é uma realidade no cotidiano de incontáveis famílias, independentemente de classe social (De Antoni, \& Koller, 2000; Ferenczi, 1992; Guerra, 2010).

Entendem-se como alternativas de enfrentamento e prevenção da violência as ações que visam qualificar e intensificar o trabalho territorial, em especial por meio do fortalecimento das Equipes de Saúde da Família juntamente com ações de articulação com as escolas da comunidade e com os dispositivos da Política de Assistência Social - Sistema Único de Assistência Social (SUAS), em especial com os Centros de Referência em Assistência Social (CRAS).

Destaca-se que a Estratégia Saúde da Família (ESF) é o modelo prioritário do SUS para a Atenção Primária a Saúde (APS). A ESF é composta por equipes multiprofissionais que são responsáveis pelo acompanhamento de um número definido de famílias, localizadas numa área geográfica delimitada, possuindo como prioridade as ações de promoção, proteção e recuperação da saúde, atuando de forma integral e continuada (Brasil, 2011c). É nesse panorama que a ESF adquire papel fundamental, especialmente por ter em sua equipe o Agente Comunitário de Saúde (ACS). Por meio deste profissional e da visita domiciliar, as manifestações da violência podem ficar mais perceptíveis. O ACS é o elo entre a equipe e a comunidade, e a visita domiciliar, além de possibilitar a compreensão da dinâmica familiar e comunitária, e permite o desenvolvimento de ações preventivas e educativas.

Acrescenta-se a importância da ampliação dos Núcleos de Apoio à Saúde da Família (NASF), pois é por meio do NASF que a Psicologia se inseriu definitivamente no campo da APS. Desse modo, a presença do psicólogo no NASF pode fortalecer e qualificar o processo de trabalho das equipes de ESF e dos ACS, ampliando as possibilidades de cuidado no território, por meio de ações de educação permanente, promoção de saúde, prevenção da violência e articulação com os demais dispositivos da rede de proteção.

Além do fortalecimento da atuação do psicólogo no campo da APS, destaca-se a necessidade de investimentos em serviços de atendimento psicossocial e psicoterapia para crianças e adolescentes vítimas de violência, por meio de práticas complementares entre psicólogos que atuam no SUS e no SUAS (Hohendorff, Habigzang, \& Koller, 2015). Outra contribuição da Psicologia no contexto da violência é a atuação junto ao sistema de justiça, na qual o psicólogo pode qualificar a tomada de decisão dos operadores do Direito, atuando como mediador entre a criança/adolescente e contexto judiciário (Eloy, 2012; Pelisoli, \& Dell'Aglio, 2014). Constata-se, portanto, que a Psicologia tem muito a contribuir, seja na promoção da qualidade de vida às famílias, na prevenção das violências, como também no atendimento às vítimas e responsabilização dos agressores.

É preciso considerar também que a notificação da violência que envolve a infância e a adolescência pode ser realizada em diversos espaços, como Conselhos Tutelares, Delegacias de Proteção a Crianças e Adolescentes, Ministério Público, Hospitais de Urgência e Emergência, Disque 100, Sistema de Vigilância de Violências e Acidentes, Sinan, entre outros. Contudo, ressalta-se que os dados apresentados neste estudo referem-se às notificações de violência registradas no Sinan-Net: violência doméstica, sexual e/ou outras, não contemplando uma articulação com as violências que chegam a outros serviços e/ ou setores.

Ressalta-se, portanto, que a não uniformização em relação aos dados epidemiológicos das violências leva a uma pulverização dos registros, fazendo com que esse fenômeno seja percebido de forma fragmentada pelos diversos setores que deveriam estar integrados com vistas ao seu enfrentamento (Moreira et al., 2013). Percebe-se, pois, que a precariedade do dimensionamento epidemiológico da violência é outro obstáculo ao enfrentamento do fenômeno (Azevedo, Guerra, 
Zweiman, \& Nishizawa, 2001). A pouca notificação ou a desarticulação dos dados ocasiona déficits na qualidade da informação, levando ao não conhecimento detalhado do fenômeno, fazendo com que as manifestações da violência se tornem praticamente invisíveis aos gestores e prejudicando o planejamento de ações e políticas públicas (Arpini et al., 2008; Assis et al., 2012; Azambuja, 2005).

Destaca-se ainda a importância da integração dos dados epidemiológicos das violências, por meio da construção de um sistema de informação universal, que inclua os registros da Saúde, Assistência Social, Segurança Pública, Disque 100 e demais instituições que se envolvem com as notificações de violência, para que seja possível dimensionar de maneira mais fidedigna e não fragmentada o perfil da violência no Brasil.

Desse modo, uma análise crítica dos aspectos abordados evidencia a importância da construção de um trabalho em rede, no qual se identifica a necessidade de superar aspectos tradicionais presentes nas práticas de saúde, como o isolamento entre as ações realizadas e o registro das informações (Garbin et al., 2015; Santos, Ipollito, \& Magalhães, 2014). Para que efetivamente as crianças e os adolescentes vivam como sujeitos de direitos, é fundamental que os profissionais ampliem suas praticas. Entre os profissionais, em especial chamamos atenção para o núcleo de Psicologia que se encontra fortemente desafiado pelos novos rumos das políticas públicas no Brasil.

Acompanhar tais desafios exige mudanças na formação dos psicólogos e sensibilidade para superar aspectos muito presentes na história da Psicologia como profissão, em especial o sigilo e o registro das informações (Conselho Federal de Psicologia, 2009). Trabalhar em equipe e tomar decisões compartilhadas deve fazer parte do processo de fortalecimento das notificações, as quais possibilitam ampliar as possibilidades no campo da proteção, atendimento e responsabilização (Faleiros, 2003; Garbin et al., 2015). Eis aqui grandes desafios que merecem importantes reflexões críticas e transformadoras.

\section{Considerações finais}

Identificou-se, por meio deste estudo, que o perfil da violência e as características das vítimas têm mantido um padrão na cidade investigada em relação ao estado do Rio Grande do Sul e demais estudos realizados no Brasil. Independentemente da porta de entrada das notificações - Saúde, Segurança Pública, Assistência Social, Disque 100 -, os dados epidemiológicos mostraram que as crianças e os adolescentes vêm sendo vítimas, sobretudo, da violência doméstica/intrafamiliar.

Portanto, é necessário avançar na utilização e problematização dos dados epidemiológicos, visto que existe um padrão que se mantém durante anos em relação às vítimas, agressores e locais de ocorrência das violências. Diante disso, entende-se como fundamental encontrar mecanismos de enfrentamento da violência que perpassam o cuidado às famílias, e fortalecer as ações de prevenção, que envolvem intervenções plurais, interdisciplinares e intersetoriais.

Conclui-se que a notificação compulsória da violência ainda é um desafio ao campo da Saúde Pública e que tal procedimento pode ser um valioso instrumento de proteção às crianças e aos adolescentes, quando integrado a outras práticas de cuidado e às Políticas Públicas direcionadas à infância, à adolescência e à família. Tal procedimento, aliado aos avanços conquistados em termos de legislação e diretos da infância e adolescência, constitui-se num importante recurso para avançar na superação da subnotificação, possibilitando maior clareza em relação ao fenômeno, fomentando investimentos direcionados adequadamente ao enfrentamento das violências.

Destarte, a relevância desta publicação está em fortalecer a visualização do fenômeno da violência, destacando a importância do instrumento de notificação, o qual exige compromisso ético dos profissionais. Tal fato possibilita reflexões para o núcleo da Psicologia, especialmente em sua interface com o campo da saúde pública.

\section{Referências}

Apostólico, M.R., Nóbrega, C.R., Guedes, R.N., Fonseca, R.M.G.S., \& Egry, E.Y. (2012). Características da violência contra a criança em uma capital brasileira. Revista Latino- Americana de Enfermagem, 20(2), 266-273. https://doi.org/10.1590/S0104-11692012000200008

Ariès, P. (1981). História social da criança e da família. Rio de Janeiro, RJ: Guanabara.

Arpini, D. M., \& Silva, M. L. (2006). A violência com crianças e adolescentes na cidade de Santa Maria. Santa Maria: Universidade Federal de Santa Maria. 
Arpini, D. M., Soares, A. C. O., Bertê, L., \& Dal Forno, C. (2008). A revelação e a notificação das situações de violência contra a infância e a adolescência. Psicologia em Revista, 14(2), 95-112. Recuperado de http://periodicos.pucminas.br/index.php/psicologiaemrevista/article/view/334

Assis, S. G. (1994). Crianças e adolescentes violentados: passado, presente e perspectivas para o futuro. Cadernos de Saúde Pública, 10 (supl 1), 126-134. https://doi.org/10.1590/S0102-311X1994000500008

Assis, S. G., Avanci, J. Q., Pesce, R. P., Pires, T. O., \& Gomes, D. L. (2012). Notificações de violência doméstica, sexual e outras violências contra crianças no Brasil. Ciência \& Saúde Coletiva, 17(9), 2305-2317. https://doi.org/10.1590/S1413-81232012000900012

Azambuja, M. P. R. (2005). Violência doméstica: reflexões sobre o agir profissional. Psicologia Ciência e Profissão, 25(1), 4-13. https://doi.org/10.1590/S1414-98932005000100002

Azevedo, M. A., Guerra, V. N. A., Zweiman, A. B., \& Nishizawa, E. (2001). Educação a distância: o combate à violência doméstica contra crianças e adolescentes: uma experiência bem sucedida. Psicologia em Estudo, 6(1), 85-89. https://doi.org/10.1590/S1413-73722001000100011

Brasil. (1990, 16 de julho). Lei No 8.069, de 13 de julho de 1990. Dispõe sobre o Estatuto da Criança e do Adolescente e dá outras providências. Diário Oficial da União.

Brasil. (2009). Ministério da Saúde. Impacto da violência na saúde das crianças e adolescentes. Brasília, DF: o autor.

Brasil. (2011c, 24 de outubro). Ministério da Saúde. Portaria No 2.488, de 21 de outubro de 2011. Aprova a Política Nacional de Atenção Básica, estabelecendo a revisão de diretrizes e normas para a organização da Atenção Básica, para a Estratégia Saúde da Família (ESF) e o Programa de Agentes Comunitários de Saúde (PACS). Diário Oficial da União.

Brasil. (2011b, 26 de janeiro). Ministério da Saúde. Portaria No 104, de 25 de janeiro de 2011. Define as terminologias adotadas em legislação nacional, conforme o disposto no Regulamento Sanitário Internacional 2005 (RSI 2005), a relação de doenças, agravos e eventos em saúde pública de notificação compulsória em todo o território nacional e estabelece fluxo, critérios, responsabilidades e atribuições aos profissionais e serviços de saúde. Diário Oficial da União.

Brasil. (2014, 9 de junho). Ministério da Saúde. Portaria No 1.271, de 6 de junho de 2014. Define a Lista Nacional de Notificação Compulsória de doenças, agravos e eventos de saúde pública nos serviços de saúde públicos e privados em todo o território nacional, nos termos do anexo, e dá outras providências. Diário Oficial da União.

Brasil. (2006). Ministério da Saúde. Secretaria de Atenção à Saúde. Prevenção do suicídio: manual dirigido a profissionais das equipes de saúde mental. Brasília, DF: o autor

Brasil. (2011a). Ministério da Saúde. Secretaria de Vigilância em Saúde. Viva: instrutivo de notificação de violência doméstica, sexual e outras violências (Série F, Comunicação e educação em saúde). Brasília, DF: o autor.

Brasil. (2008). Ministério da Saúde. Temático prevenção da violência e cultura da paz. Brasília, DF: Organização Pan-Americana da Saúde.

Brito, A. M. M., Zanetta, D. M. T., Mendonça, R. C. V., Barison, S. Z. P., \& Andrade, V. A. G. (2005). Violência doméstica contra crianças e adolescentes: estudos de um programa de intervenção. Ciência \& Saúde Coletiva, 10(1), 143-149. https://doi.org/10.1590/S1413-81232005000100021

Cezar, P. K., Rodrigues, P. M. \& Arpini, D. M. (2015) . A psicologia na estratégia de saúde da família: vivências da residência multiprofissional. Psicologia:Ciência e Profissão, 35(1), 211-224. https://doi.org/10.1590/1982-3703000012014

Conselho Federal de Psicologia. (2009). Resolução No 001, de março de 2009. Dispõe sobre a obrigatoriedade do registro documental decorrente da prestação de serviços psicológicos. Brasília, DF: o autor.

Costa, M. C. O., Carvalho, R. C., Santa Bárbara, J.F. R., Santos, C. A. S. T., Gomes, W. A., \& Sousa, H. L. (2007). O perfil da violênciacontracrianças eadolescentes, segundoregistrosdosConselhosTutelares:vítimas, agressoresemanifestações de violência. Ciência \& Saúde Coletiva, 12(5),1129-1141. https://doi.org/10.1590/S1413-81232007000500010

De Antoni, C., \& Koller, S.H. (2000). A visão sobre família entre as adolescentes que sofreram violência intrafamiliar. Estudos de Psicologia, 5(2), 347-381. https://doi.org/10.1590/S1413-294X2000000200004

Deslandes, S., Mendes, C. H. F., Lima, J. S., \& Campos, D. S. (2011). Indicadores das ações municipais para a notificação e o registro de casos de violência intrafamiliar e exploração sexual de crianças e adolescentes. Cadernos de Saúde Pública, 27(8),1633-1645. https://doi.org/10.1590/S0102-311X2011000800018

Eliachef, C. (2007). Todos vítimas? A propósito dos maus-tratos à criança. In S. Altoé (Org.), A lei e as leis: direito e psicanálise (pp. 163-172). Rio de Janeiro, RJ: Revinter. 
Eloy, C.B. (2012). A credibilidade do testemunho da criança vítima de abuso sexual no contexto judiciário. Psicologia: Ciência e Profissão, 32(1), 234-249. https://doi.org/10.1590/S1414-98932012000100017

Faleiros, E. T. S. (2003). O abuso sexual contra crianças e adolescentes: os descaminhos da denúncia. Brasília, DF: Secretaria Especial de Direitos Humanos.

Faleiros, V., \& Faleiros, E. S. (2008). Escola que protege: enfrentando a violência contra crianças e adolescentes. Brasília, DF: Ministério da Educação.

Ferenczi, S. (1992). Confusão de língua entre os adultos e a criança. In Obras completas, Psicanálise IV (A. Cabral, trad., pp. 97-106). São Paulo, SP: Martins Fontes.

Flick, U. (2009). Introdução à pesquisa qualitativa. Porto Alegre, RS: Artmed.

Galvão, V. A. B. M., \& Dimenstein, M. (2009). O protocolo de notificação da violência: entre o risco e a vulnerabilidade. Mental, 7(13). Recuperado de http://pepsic.bvsalud.org/pdf/mental/v7n13/v7n13a10.pdf

Garbin, C. A. S., Dias, I. A., Rovida, T. A. S., \& Garbin, A. J. I. (2015). Desafios do profissional de saúde na notificação da violência; obrigatoriedade, efetivação e encaminhamento. Ciência \& Saúde Coletiva, 20(6). 1879-1890. http://dx.doi.org/10.1590/1413-81232015206.13442014

Garbin, C. A. S., Garbin, A. J. I.,Moimaz, S. A. S., Saliba, O., Costa, A. C. O., \& Queiroz, A. P. D. G. (2011). Notificação de violência contra criança: conhecimento e comportamento dos profissionais de saúde. Revista Brasileira de Pesquisa em Saúde, 13(2), 17-23. https://doi.org/10.21722/rbps.v0i0.1597

Gonçalves,H.S., \&Ferreira, A.L. (2002). Anotificação da violênciaintrafamiliar contra crianças eadolescentes por profissionais desaúde. CadernosdeSaúdePública, 18(1),315-319.https://doi.org/10.1590/S0102-311X2002000100032

Gonçalves, H. S., Ferreira, A. L., \& Marques, M. (1999). Avaliação de serviço de atenção a crianças vítimas de violência domestica. Revista de Saúde Pública, 33(6), 547-553. https://doi.org/10.1590/S0034-89101999000600005

Guerra, V. N. A. (2010). Violência de pais contra filhos: a tragédia revisitada (7a ed.) São Paulo, SP: Cortez.

Habigzang, L. F., Koller, S. H., Azevedo, G. A., \& Machado, P. X. (2005). Abuso sexual infantil e dinâmica familiar: aspectos observados em processos jurídicos. Psicologia: Teoria e Pesquisa, 21(3), 341-348. https://doi.org/10.1590/S0102-37722005000300011

Hohendorff, J.V., Habigzang, L. F., \& Koller, S. H. (2015). Psicoterapia para crianças e adolescentes vítimas de violência sexual no sistema público: panorama e. alternativas de atendimento. Psicologia: Ciência e Profissão, 35(1),182-19. https://doi.org/10.1590/1982-3703000202014

Instituto Brasileiro de Geografia e Estatística - IBGE. Cidades: Rio Grande do Sul: Santa Maria. População 2014. Disponível em: http://www.cidades.ibge.gov.br/xtras/perfil.php?lang=\&codmun=431690

Kristensen, C. H., Oliveira, M. S., \& Flores, R. Z. (1999). Violência contra crianças e adolescentesna Grande Porto Alegre: Pode piorar? In Associação de Apoio à Criança e ao Adolescente (Ed.), Violência doméstica (pp. 104-117). São Leopoldo, RS: Amencar.

Laville, C. \& Dionne, J. (1999). A construção do saber: manual de metodologia da pesquisa em ciências humanas. Belo Horizonte, MG: UFMG.

Lobato, G., Moraes, C., \& Nascimento, M. (2012). Desafios da atenção à violência doméstica contra crianças e adolescentes no Programa Saúde da Família em cidade de médio porte do Estado do Rio de Janeiro, Brasil. Cadernos de Saúde Pública, 28(9), 1749-1758. https://doi.org/10.1590/S0102-311X2012000900013

Minayo, M. C. S. (2006). A inclusão da violência na agenda da saúde: trajetória histórica. Ciência \& Saúde Coletiva, 11(supl 0), 1259-1267. https://doi.org/10.1590/S1413-81232006000500015

Minayo, M. C. S. (1994). Violência social sob a perspectiva da saúde pública. Cadernos de Saúde Pública, 10(supl 1), 7-18. http://dx.doi.org/10.1590/S0102-311X1994000500002

Minayo, M. C. S., \& Souza, E. R. (1999). É possível prevenir a violência? Reflexões a partir do campo da saúde pública. Ciência \& Saúde Coletiva, 4(1), 7-23. https://doi.org/10.1590/S1413-81231999000100002

Moreira, M. I. C., Sousa, S. M. G., \& Silva, M. A. G. A. (2013). A violência intrafamiliar contra crianças e adolescentes: análise da situação e recomendações para o enfrentamento e prevenção. In S. M. G. Sousa, \& M. I. C. Moreira (Orgs.), Quebrando o silêncio: Ndisque 100 (pp. 31-70). Goiânia, GO: Cânone. 
Pelisoli, C., \& Dell'Aglio, D. D. (2014). As contribuições da psicologia para o sistema de justiça em situações de abuso sexual. Psicologia: Ciência e Profissão, 34(4), 916-930. https:// doi.org/10.1590/1982-370001032013

Pelisoli, C., Pires, J. P. M., Almeida, M. E., \& Dell'Aglio, D. D. (2010). Violência sexual contra crianças e adolescentes: dados de um serviço de referência. Temas em Psicologia, 8(1), 85-97. Recuperado de http://pepsic.bvsalud.org/scielo.php?script=sci_abstract\&pid=S1413-389X2010000100008

Rio Grande do Sul. (2014). Secretaria Estadual da Saúde. Tabnet: Dados epidemiológicos - SINAN: violência doméstica, sexual elou outras, RS, 2009-2013. Porto Alegre, RS: o autor. Recuperado de http://200.198.173.164/scripts/tabcgi.exe?snet/violencianet

Santos, B. R. (2014). Por uma escuta da criança e do adolescente social e culturalmente contextualizada: concepções de infância e de adolescência, universalidade de direitos e respeito às diversidades.In B. R. Santos, I. B. Gonçalves (Coords.), Escuta de crianças e adolescentes em situação de violência sexual: aspectos teóricos e metodológicos: guia para capacitação em depoimento especial de crianças e adolescentes (pp. 27-42). Brasília, DF: EdUCB.

Santos, B. R., Ippolito, R. \& Magalhães, M .L. (2014). Políticas públicas, rede de proteção e os programas e serviços voltados para crianças e adolescentes em situação de violência sexual. In B. R. Santos, \& I. B Gonçalves (Coords.), Escuta de crianças e adolescentes em situação de violência sexual: aspectos teóricos e metodológicos: guia para capacitação em depoimento especial de crianças e adolescentes (pp. 113-129). Brasília, DF: EdUCB.

Senado Federal. (1988). Constituição da República Federativa do Brasil. Brasília, DF: o autor.

Veloso, M. M. X., Magalhães, C. M. C., Dell'Aglio, D. D., Cabral, I. R., \& Gomes, M. M. (2013). Notificação da violência como estratégia de vigilância em saúde: perfil de uma metrópole do Brasil. Ciência \& Saúde Coletiva, 18(5), 1263-1272. https://doi.org/10.1590/S1413-81232013000500011

Waiselfisz, J. J. (2012). Mapa da Violência 2012: crianças e adolescentes do Brasil. Rio de Janeiro, RJ: Cebela.

Zanatta, E. A., Pai, D. D., Resta, D. G., Argenta, C., \& Motta, M. G. C. (2012). Caracterização das notificações de violência contra adolescentes. Enfermagem em Foco, 3(4), 165-168. https:// doi.org/10.21675/2357-707X.2012.v3.n4.376

\section{Pâmela Kurtz Cezar}

Mestrado com ênfase em Psicologia da Saúde pelo Programa de Pós-Graduação em Psicologia da Universidade Federal de Santa Maria, RS, Brasil.

E-mail: pamelakurtz@gmail.com

\section{Dorian Mônica Arpini}

Doutora em Psicologia Social pela Pontifícia Universidade Católica de São Paulo, SP, Brasil.

E-mail: monica.arpini@gmail.com

Everley Rosane Goetz

Doutora em Psicologia pela Universidade Federal de Santa Catarina, SC, Brasil.

E-mail: eve.goetz35@gmail.com

Endereço para envio de correspondência:

Universidade Federal de Santa Maria. Centro de Ciências Sociais e Humanas. Programa de Pós-graduação em Psicologia - Avenida Roraima no 1000, prédio 74B, sala 3208. Cidade Universitária.

Bairro Camobi. CEP 97105-900 - Santa Maria, RS - Brasil.

Recebido 13/06/2015

Reformulação 08/09/2016

Aprovado 14/02/2017

Received 06-13/2015

Reformulated $05 / 10 / 2016$

Approved 02/14/2017 
Recebido 13/06/2015

Reformulado 09/08/2016

Aceptado 14/02/2017

Como citar: Cezar, P. K., Arpini, D. M., \& Goetz, E. R. (2017). Registros de notificação compulsória de violência envolvendo crianças e adolescentes. Psicologia: Ciência e Profissão, 37(2), 432-445. https://doi.org/10.1590/1982-370301942015

How to cite: Cezar, P. K., Arpini, D. M., \& Goetz, E. R. (2017). Records of compulsory notification of violence against children and adolescents. Psicologia:CiênciaeProfissão, 37(2), 432-445.https://doi.org/10.1590/1982-370301942015

Cómo citar: Cezar, P. K., Arpini, D. M., \& Goetz, E. R. (2017). Registros de notificación

obligatoria de violencia contra niños y adolescentes. Psicologia: Ciência e Profissão, 37(2), 432-445.

https://doi.org/10.1590/1982-370301942015 